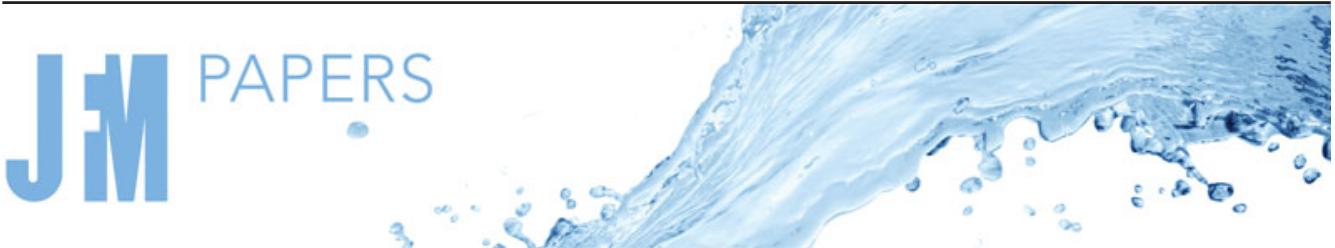

\section{Macrotransport theory for diffusiophoretic colloids and chemotactic microorganisms}

\author{
Henry C.W. Chu ${ }^{1, \dagger}$, Stephen Garoff ${ }^{2}$, Robert D. Tilton ${ }^{3}$ and Aditya S. Khair ${ }^{4}$ \\ ${ }^{1}$ Department of Chemical Engineering, University of Florida, Gainesville, FL 32611, USA \\ ${ }^{2}$ Department of Physics and Center for Complex Fluids Engineering, Carnegie Mellon University, \\ Pittsburgh, PA 15213, USA \\ ${ }^{3}$ Department of Chemical Engineering, Department of Biomedical Engineering, and Center for Complex \\ Fluids Engineering, Carnegie Mellon University, Pittsburgh, PA 15213, USA \\ ${ }^{4}$ Department of Chemical Engineering and Center for Complex Fluids Engineering, Carnegie Mellon \\ University, Pittsburgh, PA 15213, USA
}

(Received 9 November 2020; revised 3 March 2021; accepted 2 April 2021)

We conduct an asymptotic analysis to derive a macrotransport equation for the long-time transport of a chemotactic/diffusiophoretic colloidal species in a uniform circular tube under a steady, laminar, pressure-driven flow and transient solute gradient. The solute gradient drives a 'log-sensing' advective flux of the colloidal species, which competes with Taylor dispersion due to the hydrodynamic flow. We demonstrate excellent agreement between the macrotransport equation and direct numerical solution of the full advection-diffusion equation for the colloidal species transport. In addition to its accuracy, the macrotransport equation requires $O\left(10^{3}\right)$ times less computational runtime than direct numerical solution of the advection-diffusion equation. Via scaling arguments, we identify three regimes of the colloidal species macrotransport, which span from chemotactic/diffusiophoretic-dominated macrotransport to the familiar Taylor dispersion regime, where macrotransport is dominated by the hydrodynamic flow. Finally, we discuss generalization of the macrotransport equation to channels of arbitrary (but constant) cross-section and to incorporate more sophisticated models of chemotactic fluxes. The macrotransport framework developed here will broaden the scope of designing chemotactic/diffusiophoretic transport systems by elucidating the interplay of macrotransport due to chemotaxis/diffusiophoresis and hydrodynamic flow.

Key words: mixing and dispersion, colloids, microfluidics

$\dagger$ Email address for correspondence: h.chu@ufl.edu

(C) The Author(s), 2021. Published by Cambridge University Press. This is an Open Access article, distributed under the terms of the Creative Commons Attribution licence (http://creativecommons.org/ licenses/by/4.0/), which permits unrestricted re-use, distribution, and reproduction in any medium, provided the original work is properly cited. 


\section{Introduction}

Deterministic motion of a colloidal-scale species can be induced by the concentration gradient of a surrounding solute. For charged colloids in electrolyte solutions, the concentration gradient of the electrolyte causes motion known as diffusiophoresis (Anderson 1989; Velegol et al. 2016; Marbach \& Bocquet 2019). Diffusiophoresis is an electrokinetic phenomenon, comprising a chemiphoretic component due to the osmotic pressure gradient developed along the colloid surface, and an electrophoretic component due to the junction potential generated by the diffusion of ions with different diffusivities. Prieve et al. (1984) derived the drift velocity of a diffusiophoretic colloid, $\boldsymbol{u}=M \nabla \log S$, which was confirmed experimentally (Staffeld \& Quinn 1989; Abecassis et al. 2008; Palacci et al. 2010, 2012; Kar et al. 2015; Banerjee et al. 2016; Shi et al. 2016; Shin et al. 2016; Ault et al. 2017; Shin et al. 2017; Ault, Shin \& Stone 2018). The diffusiophoretic velocity relates to the gradient of the logarithm of the solute concentration $S$ via the diffusiophoretic mobility $M$, which has been termed a 'log-sensing' response (Palacci et al. 2012). The mobility encompasses information about the colloid and the solute, including the colloid surface potential and ionic diffusivities. A positive and a negative $M$ correspond to the colloids driven up and down the solute gradient, respectively. Since the solute concentration in a system is often inhomogeneous, diffusiophoresis plays a role in numerous natural phenomena and applications such as mineral replacement reactions, drug delivery and enhanced oil recovery (Velegol et al. 2016; Marbach \& Bocquet 2019).

While diffusiophoresis is a physico-chemical phenomenon, perhaps surprisingly it shares the same log-sensing relation with a biological phenomenon: chemotaxis (Keller \& Segel 1971; Brown \& Berg 1974; Kalinin et al. 2009; Marbach \& Bocquet 2019). Chemotaxis refers to microorganisms utilizing their transmembrane chemoreceptors to detect the surrounding solute gradient, along which they perform deterministic motion (Engelmann 1881; Adler 1966; Brown \& Berg 1974; Parkinson \& Kofoid 1992; Eisenbach et al. 2004; Wadhams \& Armitage 2004). A widely studied example is Escherichia coli. In an inhomogeneous solute field, the bacteria sense the solute gradient in a temporal fashion and use that information to modulate the probability of their run-and-tumble events. Runs are extended in favourable directions, resulting in a net movement of the bacteria up/down the gradient of the solute (attractant/repellent) (Brown \& Berg 1974; Wu et al. 2006). Chemotaxis is central to phenomena such as biofilm formation (Eisenbach et al. 2004) and has been utilized in bioremediation including aquifer restoration (Ford \& Harvey 2007; Adadevoh et al. 2017). In chemotactic log-sensing, $M$ is the chemotactic sensitivity. Velocity relations other than log-sensing have been proposed to capture various hallmarks of chemotaxis in more general regimes (Lapidus \& Schiller 1976; Segel 1977; Rivero et al. 1989; Tindall et al. 2008; Menolascina et al. 2017; Salek et al. 2019). Recent studies also suggest that diffusiophoresis could contribute to the movement of living colloidal species in addition to chemotaxis (Marbach \& Bocquet 2019; Sear 2019).

To model the spatio-temporal evolution of a population of chemotactic/diffusiophoretic colloidal species (which we will refer to as 'colloids' in the rest of this article, for brevity), one must solve the coupled solute and colloid advection-diffusion-reaction equations (Lapidus \& Schiller 1976; Rivero-Hudec \& Lauffenburger 1986; Staffeld \& Quinn 1989; Ford \& Cummings 1992; Marx \& Aitken 2000; Abecassis et al. 2008; Tindall et al. 2008; Palacci et al. 2010, 2012; Kar et al. 2015; Banerjee et al. 2016; Shi et al. 2016; Shin et al. 2016; Ault et al. 2017, 2018; Shin et al. 2017; Raynal et al. 2018; Raynal \& Volk 2019; Shim, Stone \& Ford 2019; Chu et al. 2020a). The evolving solute gradient induces a chemical-driven chemotactic/diffusiophoretic drift velocity which contributes to the advective flux of the colloids, in addition to that due to any imposed hydrodynamic 


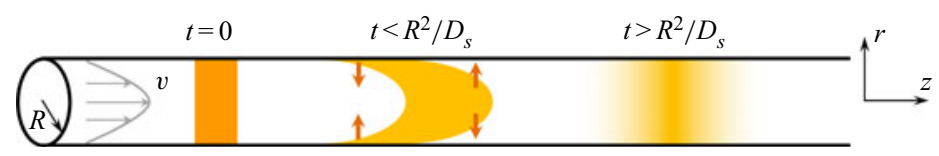

Figure 1. Illustration of Taylor hydrodynamic dispersion.

flow, $v$, such as blood flows in diffusiophoretic colloidal drug delivery and interstitial fluid flows in chemotactic bioremediation of aquifers. Reactions of colloids refer to an increase (source) or decrease (sink) in the total amount of the colloids, e.g. death of bacteria (Servais, Billen \& Rego 1985; Golding et al. 1998; Tindall et al. 2008). In general, chemotactic/diffusiophoretic colloid transport occurs in two or higher spatial dimensions, for example, in a microchannel. Predicting the transport via direct numerical simulations is costly especially at long times, since the shortest time scale where the transport occurs has to be resolved. For instance, for chemotaxis/diffusiophoresis in a hydrodynamic flow in a long thin channel of radius $R$, where the colloid diffusivity $D_{c}$ is typically smaller or comparable to the solute diffusivity $D_{s}$ (Ford \& Lauffenburger 1991; Lewus \& Ford 2001; Tindall et al. 2008; Cussler 2009; Shin et al. 2016; Shim et al. 2019), the shortest transport time scale is the solute radial diffusion time $R^{2} / D_{s}$. This is much smaller than the solute(colloid) convective time $L / \bar{v}$ along the length of the channel $L$ ( $v$ is the axial component of $v$ and overbar denotes a cross-sectional average). Nevertheless, one can leverage this separation of time scales to construct asymptotic schemes to predict the solute and colloid transport.

To illustrate the idea, let us first focus on the solute transport in the absence of colloids, as shown in figure 1 . The hydrodynamic flow induces concentration gradients of the solute in the radial direction for times shorter than $R^{2} / D_{s}$. However, diffusion acts to smooth these gradients. At times longer than $R^{2} / D_{s}$, where the solute has fully sampled the velocity variations across the cross-section of the channel, its concentration no longer varies in the radial direction: only axial gradients persist. Hence, the transport becomes one-dimensional. Taylor (1953) originally made this brilliant physical observation and subsequently proposed and experimentally verified that the two-dimensional transport equation for the solute concentration can be reduced to a one-dimensional 'macrotransport' equation for the cross-sectional averaged solute concentration. Specifically, the averaged concentration field translates with the mean speed of the hydrodynamic flow and undergoes an enhanced axial diffusion, or Taylor dispersion (Aris 1956; Brenner \& Edwards 1993), due to the coupling between axial convection and radial diffusion.

In this work, we follow Taylor's approach (Taylor 1953) to derive a macrotransport equation for a chemotactic/diffusiophoretic colloidal species under hydrodynamic flows and transient solute gradients. A key idea is that, because radial solute gradients are homogenized at long times, chemical-driven chemotactic/diffusiophoretic fluxes in the radial direction can be ignored in the colloid macrotransport. Axial solute gradients and thus chemical-driven fluxes in the axial direction may still be present, however, and they are captured in the colloid macrotransport. The rest of this article is outlined as follows. In $\S 2$, we formulate the problem by deriving a macrotransport (averaged) equation for the two-dimensional, advection-diffusion-reaction transport of a chemotactic/diffusiophoretic colloidal species in a uniform, circular channel. We define three flow regimes, from weak to strong hydrodynamic flow strength, where the macrotransport equation is applicable. In $\S 3$, we verify the macrotransport equation by comparing its predicted colloid transport with that from direct numerical simulation of the 


\section{H.C.W. Chu, S. Garoff, R.D. Tilton and A.S. Khair}

two-dimensional equation. Comparisons are conducted for all three aforementioned flow regimes as well as for a non-unity colloid-to-solute diffusivity ratio, suitable for modelling chemotaxis and diffusiophoresis. The implementation of the macrotransport equation to a non-circular channel and non-log-sensing models of chemical-driven transport is also elucidated. In $\S 4$, we summarize this study and offer ideas for future work.

\section{Diffusiophoretic/chemotactic macrotransport model}

\subsection{Mathematical formulation}

We consider diffusiophoresis/chemotaxis of a colloidal species due to a surrounding solute gradient in an incompressible, unidirectional hydrodynamic flow $v$ inside a uniform, circular channel of radius $R$ (figure 2). The channel wall is impenetrable to the solute and the colloid. The steady hydrodynamic flow $v(r)$ is directed along the axial direction $z$ and may vary in the radial direction $r$. The colloid $C(r, z, t)$ and solute $S(r, z, t)$ concentration fields are symmetrically distributed about the channel centreline, and may vary in the radial and axial directions, and in time $t$. For $C \ll S$, which is common for colloidal or bacterial suspensions containing molecular solutes, the influence of the evolution of $C$ on $S$ is negligible (Lapidus \& Schiller 1976; Rivero-Hudec \& Lauffenburger 1986; Staffeld \& Quinn 1989; Ford \& Cummings 1992; Marx \& Aitken 2000; Abecassis et al. 2008; Tindall et al. 2008; Palacci et al. 2010, 2012; Kar et al. 2015; Banerjee et al. 2016; Shi et al. 2016; Shin et al. 2016; Ault et al. 2017, 2018; Peraud et al. 2017; Shin et al. 2017; Balu \& Khair 2018; Raynal et al. 2018; Raynal \& Volk 2019; Shim et al. 2019; Chu et al. 2020a). The advection-diffusion transport of the solute is governed by

$$
\frac{\partial S}{\partial t}+v \frac{\partial S}{\partial z}=\frac{D_{s}}{r} \frac{\partial}{\partial r}\left(r \frac{\partial S}{\partial r}\right)+D_{s} \frac{\partial^{2} S}{\partial z^{2}},
$$

where $D_{s}$ is the constant, intrinsic solute diffusivity. The hydrodynamic flow causes solute concentration gradients in the radial direction for $t<R^{2} / D_{s}$. However, diffusion acts to smooth these gradients. Following Taylor's and Aris' (Aris 1956) analyses, subsequent studies (Bailey \& Gogarty 1962; Gill \& Sankarasubramanian 1970; Ng \& Rudraiah 2008) showed that, at times larger than the solute radial diffusive time $t \geq R^{2} / D_{s}$, variations in the solute concentration across the channel cross-section have been smeared out, and the macrotransport equation for the cross-sectional averaged solute concentration, $\bar{S}(z, t)$, is

$$
\frac{\partial \bar{S}}{\partial t}+\bar{v} \frac{\partial \bar{S}}{\partial z}=\left(D_{s}+\frac{\bar{v}^{2} R^{2}}{48 D_{s}}\right) \frac{\partial^{2} \bar{S}}{\partial z^{2}} \quad \text { for } t \geq R^{2} / D_{s},
$$

where the cross-sectional average is $\overline{(\cdot)}=\int_{0}^{2 \pi} \int_{0}^{R}(\cdot) r \mathrm{~d} r \mathrm{~d} \theta / \pi R^{2}$ with $\theta$ being the azimuth, and the second term in the bracket is an enhanced axial diffusion, or Taylor dispersion (Taylor 1953; Aris 1956; Brenner \& Edwards 1993), owing to the coupling between axial convection and radial diffusion.

The evolving solute gradient induces a chemical-driven flow $\boldsymbol{u}(S)$ of the diffusiophoretic/chemotactic colloids. The solute gradient also induces a slip flow adjacent to the channel walls, known as diffusioosmosis. However, the effect of diffusioosmosis on the solute and colloid transport is negligible to the leading order of the aspect ratio of the channel (Ault et al. 2018) and thus it is ignored in the present study. As discussed in $\S 1$, an important feature in common between diffusiophoresis and chemotaxis is the $\log$-sensing chemical flow response, $\boldsymbol{u}=M \nabla \log S$. Note, however, that the present derivation is not limited to any particular form of the chemical flow, and thus we keep the 


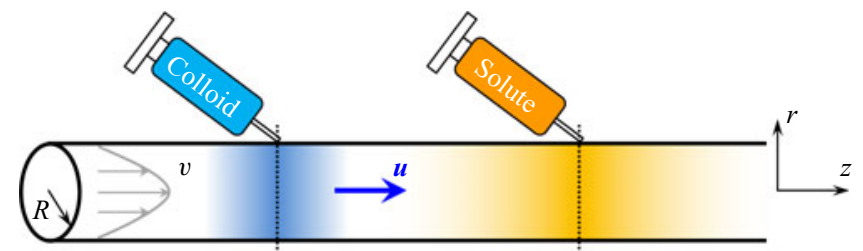

Figure 2. A diffusiophoretic/chemotactic colloidal species in a solute gradient inside a uniform, circular channel of radius $R$ under an incompressible, unidirectional hydrodynamic flow $v$. The solute gradient induces a chemical-driven flow $\boldsymbol{u}$ of the colloids via diffusiophoresis/chemotaxis, where the colloids can be attracted (as shown) or repelled.

general notation $\boldsymbol{u}$. In contrast to the hydrodynamic flow $v$, the chemical flow is usually compressible $(\boldsymbol{\nabla} \cdot \boldsymbol{u} \neq 0)$ due to the spatio-temporal variation of the solute concentration. Under the chemical and hydrodynamic flow, the advection-diffusion-reaction equation for the diffusiophoretic/chemotactic colloids is

$$
\frac{\partial C}{\partial t}+v \frac{\partial C}{\partial z}+\frac{1}{r} \frac{\partial\left(r u_{r} C\right)}{\partial r}+\frac{\partial\left(u_{z} C\right)}{\partial z}=\frac{D_{c}}{r} \frac{\partial}{\partial r}\left(r \frac{\partial C}{\partial r}\right)+D_{c} \frac{\partial^{2} C}{\partial z^{2}}-\Gamma C,
$$

where $u_{r}(r, z, t)$ and $u_{z}(r, z, t)$ are the radial and axial components of the chemical flow, respectively. In diffusiophoresis, $D_{c}$ is the constant, intrinsic diffusivity of the colloid (Staffeld \& Quinn 1989; Abecassis et al. 2008; Palacci et al. 2010, 2012; Kar et al. 2015; Banerjee et al. 2016; Shi et al. 2016; Shin et al. 2016; Ault et al. 2017, 2018; Shin et al. 2017; Raynal et al. 2018; Raynal \& Volk 2019; Chu et al. 2020a). In chemotaxis, $D_{c}$ is the random motility of the microorganism. Since a run of a bacterium typically last for approximately a second before being interrupted by a rapid [(0.1) s] tumble and subsequent change its direction (Brown \& Berg 1974; Ford \& Lauffenburger 1991; Wu et al. 2006), microorganism motility is random and can be interpreted as a diffusivity on times longer than the runtime, which is of the order of seconds. Note that such an interpretation is valid for the macrotransport equation which applies for $t \geq R^{2} / D_{c}$. For instance, in a typical microfluidic setting for bacteria where $R=10^{-4} \mathrm{~m}$ and $D_{c}=10^{-9} \mathrm{~m}^{2} \mathrm{~s}^{-1}, R^{2} / D_{c}=10 \mathrm{~s}$ is longer than the correlation time of the bacteria run-and-tumble motion. On a different note, $D_{c}$ may generally depend on the solute concentration and gradient. It is taken as a constant here as in many prior studies (see a comprehensive review by Tindall et al. 2008) and was justified for a shallow spatial and temporal gradient (Ford \& Lauffenburger 1991). Regarding the reaction term $\Gamma C$, in chemotaxis it represents the death of microorganisms due to biological cycles or toxic environments (Servais et al. 1985; Golding et al. 1998; Tindall et al. 2008). The decay rate $\Gamma$ is taken as a constant here.

Our goal is to derive an averaged, or macrotransport, equation from (2.3), suitable for probing the cross-sectionally averaged colloid concentration at long times, $t \geq R^{2} / D_{c}$. For typical diffusiophoresis/chemotaxis systems where $D_{c} / D_{s} \leq 1$ (Ford \& Lauffenburger 1991; Lewus \& Ford 2001; Tindall et al. 2008; Cussler 2009; Shin et al. 2016; Shim et al. 2019), the long-time condition for the solute is automatically satisfied so long as that for the colloid is met. Recall that for $t \geq R^{2} / D_{s}$ radial solute gradients have been homogenized by the hydrodynamic flow. This justifies dropping the radial chemical flow $u_{r}$ in (2.3) and the axial chemical flow $u_{z}$ varies only in $z$. We further write the colloid concentration and the hydrodynamic flow in terms of their cross-sectional averages (overbar) and variations, or fluctuations, therefrom (prime): $C(r, z, t)=\bar{C}(z, t)+C^{\prime}(r, z, t)$ and $v(r)=\bar{v}+v^{\prime}(r)$. 
Equation (2.3) becomes

$$
\begin{aligned}
& \frac{\partial\left(\bar{C}+C^{\prime}\right)}{\partial t}+\left(\bar{v}+v^{\prime}\right) \frac{\partial\left(\bar{C}+C^{\prime}\right)}{\partial z}+\frac{\partial\left(\bar{u}_{z} \bar{C}\right)}{\partial z}=\frac{D_{c}}{r} \frac{\partial}{\partial r}\left(r \frac{\partial C^{\prime}}{\partial r}\right) \\
& +D_{c} \frac{\partial^{2}\left(\bar{C}+C^{\prime}\right)}{\partial z^{2}}-\Gamma\left(\bar{C}+C^{\prime}\right) .
\end{aligned}
$$

The cross-sectional average of (2.4) is

$$
\frac{\partial \bar{C}}{\partial t}+\bar{v} \frac{\partial \bar{C}}{\partial z}+\overline{v^{\prime} \frac{\partial C^{\prime}}{\partial z}}+\frac{\partial\left(\bar{u}_{z} \bar{C}\right)}{\partial z}=D_{c} \frac{\partial^{2} \bar{C}}{\partial z^{2}}-\Gamma \bar{C} .
$$

To solve for $C^{\prime}$, we subtract (2.5) from (2.4) and invoke two assumptions following Taylor's classical analysis (Taylor 1953; Aris 1956; Brenner \& Edwards 1993): namely, (i) $C^{\prime} \ll \bar{C}$, and (ii) the contribution to the colloid transport by the axial diffusion is small relative to the radial diffusion at long times, $t \geq R^{2} / D_{c}$. The governing equation of $C^{\prime}$ is thus obtained as

$$
v^{\prime} \frac{\partial \bar{C}}{\partial z}=\frac{D_{c}}{r} \frac{\partial}{\partial r}\left(r \frac{\partial C^{\prime}}{\partial r}\right)-\Gamma C^{\prime} .
$$

Valdes-Parada et al. (2009) presented a similar equation to (2.6) in the development of a macrotransport theory for non-decaying $(\Gamma=0)$ chemotactic bacteria. In their equation, there is an additional term associated with 'chemotactic dispersion', that is, the enhancement or reduction of the axial diffusion of colloids due to the radial solute gradient. However, above, we have justified that such an effect can be ignored for $t \geq$ $R^{2} / D_{s}$.

Subramanian \& Gill (1974), who studied the dispersion of a decaying species in the absence of chemical flows, pointed out that (2.6) should not be used to determine the dispersion due to species decay, as was erroneously done by others (Gupta \& Gupta 1972; Vidyanidhi \& Murty 1976). They conducted a separate analysis, showing exactly that the effect of species decay only manifests in the $\Gamma \bar{C}$ term in (2.5) without any additional dispersion. In other words, $\Gamma C^{\prime}$ can be dropped from (2.6). The resulting equation can be integrated twice to obtain an expression for $C^{\prime}$ which, upon multiplying with $v^{\prime}$ and averaging the product, gives

$$
\overline{v^{\prime} C^{\prime}}=-D_{c, D i s} \frac{\partial \bar{C}}{\partial z},
$$

where the dispersivity of the colloid is defined as

$$
D_{c, \text { Dis }}=-\frac{2}{R^{2}} \int_{0}^{R}\left[\frac{v^{\prime}}{D_{c}} \int_{0}^{r} \frac{1}{r_{1}}\left(\int_{r_{1}}^{R} v^{\prime}\left(r_{2}\right) r_{2} \mathrm{~d} r_{2}\right) \mathrm{d} r_{1}\right] r \mathrm{~d} r,
$$

in which $r_{1}$ and $r_{2}$ are dummy variables. The dispersivity $D_{c, \text { Dis }}$ can be determined for a given hydrodynamic flow $v$. In the present case for a steady, pressure-driven laminar flow in a uniform circular tube, we recover Taylor's result $D_{c, \text { Dis }}=\bar{v}^{2} R^{2} / 48 D_{c}$ (Taylor 1953) for $v(r)=-\Delta P R^{2}\left(1-r^{2} / R^{2}\right) / 4 \eta, \bar{v}=-\Delta P R^{2} / 8 \eta, v^{\prime}(r)=-\Delta P R^{2}\left(1 / 2-r^{2} / R^{2}\right) / 4 \eta$, where $\Delta P$ is the applied pressure gradient and $\eta$ is the solvent viscosity. On recognizing 
that $\partial\left(\overline{v^{\prime} C^{\prime}}\right) / \partial z=\overline{v^{\prime} \partial C^{\prime} / \partial z},(2.7)$ gives

$$
\overline{v^{\prime} \frac{\partial C^{\prime}}{\partial z}}=-D_{c, D i s} \frac{\partial^{2} \bar{C}}{\partial z^{2}}
$$

Upon substituting (2.9) into (2.5), finally we obtain

$$
\frac{\partial \bar{C}}{\partial t}+\bar{v} \frac{\partial \bar{C}}{\partial z}+\frac{\partial\left(\bar{u}_{z} \bar{C}\right)}{\partial z}=\left(D_{c}+\frac{\bar{v}^{2} R^{2}}{48 D_{c}}\right) \frac{\partial^{2} \bar{C}}{\partial z^{2}}-\Gamma \bar{C} \quad \text { for } t \geq R^{2} / D_{c} .
$$

Equation (2.10) is a key result of this work: it represents a macrotransport equation for a diffusiophoretic/chemotactic colloidal species under a hydrodynamic flow and evolving solute gradient. Hydrodynamic flow contributes to dispersion via the dispersivity $D_{c, \text { Dis }}$, the second term in the bracket on the right-hand side of (2.10). We reiterate that by dispersion we mean the enhanced axial diffusion due to the coupling of axial convection and radial diffusion. Thus, chemical flow does not cause dispersion at long times but it does contribute to colloid advection and spreading, i.e. macrotransport, via the term $\partial\left(\bar{u}_{z} \bar{C}\right) / \partial z$. This term is in turn influenced by the action of the hydrodynamic flow on the solute gradient.

Equation (2.10) reduces to classical macrotransport equations in limiting cases. For instance, when there is no solute gradient, $\partial\left(\bar{u}_{z} \bar{C}\right) / \partial z$ vanishes and (2.10) recovers the result of Subramanian \& Gill (1974), where colloid diffusion is solely governed by the sum of the colloid intrinsic diffusivity/motility and hydrodynamic dispersion. Further, when there is no solute gradient and the colloid is non-decaying, $\partial\left(\bar{u}_{z} \bar{C}\right) / \partial z$ and $\Gamma \bar{C}$ vanish, and (2.10) recovers Taylor's and Aris' classical result (Taylor 1953; Aris 1956). Equation (2.10) is valid formally for $t \geq R^{2} / D_{c}$ (Bailey \& Gogarty 1962; Gill \& Sankarasubramanian 1970; Ng \& Rudraiah 2008). Thus, for most diffusiophoresis/chemotaxis systems where $D_{c} / D_{s} \leq 1$ (Ford \& Lauffenburger 1991; Lewus \& Ford 2001; Tindall et al. 2008; Cussler 2009; Shin et al. 2016; Shim et al. 2019), (2.10) is applicable for $t \geq R^{2} / D_{c} \geq$ $R^{2} / D_{s}$, whereas for cases where $D_{c} / D_{s} \geq 1$, (2.10) is applicable for $t \geq R^{2} / D_{s} \geq R^{2} / D_{c}$. A typical value of $R^{2} / D_{c}=10 \mathrm{~s}$ for chemotaxis is noted in the previous paragraph; for diffusiophoresis, $R=10^{-5} \mathrm{~m}$ and $D_{c} \leq 10^{-11} \mathrm{~m}^{2} \mathrm{~s}^{-1}, R^{2} / D_{c} \geq 10 \mathrm{~s}$ is the regime of major interest in common diffusiophoretic systems (Shin et al. 2016). We remark that (2.10) is general to any initial distributions of the solute and the colloid, such as a Gaussian or a spike. In $\S 3.5$, we will discuss the generalization of (2.10) to other models of the chemical flow and channels of arbitrary but uniform cross-sections.

\subsection{Hydrodynamic flow regimes of macrotransport}

Probstein (2003) characterized three different hydrodynamic flow regimes of the original Taylor-Aris solute macrotransport equation. To prepare for testing the present macrotransport theory in these regimes in the next section, below we recapitulate Probstein's analysis and extend it to the present colloidal species macrotransport.

To facilitate the discussion, the original Taylor-Aris solute macrotransport (2.2) is normalized using the following scales,

$$
\left.\begin{array}{l}
\hat{t}=\frac{t}{R^{2} / D_{s}}, \quad \hat{r}=\frac{r}{R}, \quad \hat{z}=\frac{z}{L}, \quad \hat{u}_{z}=\frac{u_{z}}{\bar{v}}, \quad \hat{u}_{r}=\frac{u_{r}}{\bar{v}} \frac{L}{R}, \\
\hat{\Gamma}=\frac{\Gamma}{D_{s} / R^{2}}, \quad \hat{C}=\frac{C}{C_{0}}, \quad \hat{S}=\frac{S}{S_{0}}, \quad \epsilon=\frac{R}{L}, \quad P e=\frac{\bar{v} R}{D_{s}},
\end{array}\right\}
$$

where $L$ and $\epsilon<1$ is the length and aspect ratio of the channel, respectively; $C_{0}$ and $S_{0}$ are the characteristic colloid and solute concentration; and $P e$ is the Péclet number which 
describes the relative importance of hydrodynamic convection of solute to solute intrinsic diffusivity. The normalized solute macrotransport equation reads

$$
\frac{\partial \hat{\bar{S}}}{\partial \hat{t}}+\epsilon P e \frac{\partial \hat{\bar{S}}}{\partial \hat{z}}=\left(\epsilon^{2}+\epsilon^{2} \frac{P e^{2}}{48}\right) \frac{\partial^{2} \hat{\bar{S}}}{\partial \hat{z}^{2}} .
$$

The first regime defined by Probstein is the Taylor regime, where convection dominates dispersion $\left(\epsilon P e \gg \epsilon^{2} P e^{2} / 48\right)$ and dispersion dominates intrinsic diffusion $\left(\epsilon^{2} P e^{2} / 48 \gg\right.$ $\epsilon^{2}$ ). This sets the range of $P e$ as $48 / \epsilon \gg P e \gg \sqrt{48}$. The second one is the convective axial diffusion regime, the opposite limit to the Taylor regime, where convection dominates intrinsic diffusion $\left(\epsilon P e \gg \epsilon^{2}\right)$ and the latter dominates dispersion $\left(\epsilon^{2} \gg \epsilon^{2} P e^{2} / 48\right)$. This gives $48 / \epsilon \gg P e \ll \sqrt{48}$. In between the Taylor and the convective axial diffusion regimes is the Taylor-Aris regime where convection dominates dispersion $(\epsilon P) \gg$ $\left.\epsilon^{2} P e^{2} / 48\right)$ while dispersion is comparable to intrinsic diffusion $\left(\epsilon^{2} P e^{2} / 48 \sim \epsilon^{2}\right)$. This gives $P e$ as 48/ $\epsilon \gg P e$ and $P e$ should be between $P e \gg \sqrt{48}$ and $P e \ll \sqrt{48}$. For example, $\epsilon=5 \times 10^{-4}$ and $P e=10$ would satisfy these conditions for the Taylor-Aris regime.

Here, we extend Probstein's analysis to the present colloid macrotransport (2.10). The normalized colloid macrotransport equation reads,

$$
\frac{\partial \hat{\bar{C}}}{\partial \hat{t}}+\epsilon P e \frac{\partial \hat{\bar{C}}}{\partial \hat{z}}+\epsilon P e \frac{\partial\left(\hat{\bar{u}}_{z} \hat{\bar{C}}\right)}{\partial \hat{z}}=\left(\epsilon^{2} \frac{D_{c}}{D_{s}}+\epsilon^{2} \frac{D_{s}}{D_{c}} \frac{P e^{2}}{48}\right) \frac{\partial^{2} \hat{\bar{C}}}{\partial \hat{z}^{2}}-\hat{\Gamma} \hat{\bar{C}} .
$$

The range of $P e$ for each regime is obtained as follows. Convective axial diffusion regime: $(48 / \epsilon)\left(D_{c} / D_{s}\right) \gg P e \ll\left(D_{c} / D_{s}\right) \sqrt{48}$; Taylor-Aris regime: $(48 / \epsilon)\left(D_{c} / D_{s}\right) \gg P e$ for $P e$ between $P e \gg\left(D_{c} / D_{s}\right) \sqrt{48}$ and $P e \ll\left(D_{c} / D_{s}\right) \sqrt{48}$; Taylor regime: $(48 / \epsilon)\left(D_{c} / D_{s}\right) \gg$ $P e \gg\left(D_{c} / D_{s}\right) \sqrt{48}$. Since the colloid macrotransport requires coupling to the solute macrotransport, their conditions for $P e$ have to be considered together. Focusing on $D_{c} / D_{s} \leq 1$, this sets the following conditions for the present macrotransport framework:

$$
\left.\begin{array}{c}
\text { Convective axial diffusion : } \frac{48}{\epsilon} \frac{D_{c}}{D_{s}} \gg P e \ll \frac{D_{c}}{D_{s}} \sqrt{48}, \\
\text {-Aris }: \frac{48}{\epsilon} \frac{D_{c}}{D_{s}} \gg P e, \text { between } P e \gg \sqrt{48} \text { and } P e \ll \frac{D_{c}}{D_{s}} \sqrt{48}, \\
\text { Taylor }: \frac{48}{\epsilon} \frac{D_{c}}{D_{s}} \gg P e \gg \sqrt{48} .
\end{array}\right\}
$$

The convective axial diffusion, Taylor-Aris and Taylor regime can be interpreted as weak, intermediate and strong hydrodynamic flow regimes, respectively. In the next section, we compare the colloid transport in these regimes predicted from the macrotransport equations $((2.2)$ and (2.10)) with that from direct numerical simulation of the two-dimensional transport equations ((2.1) and (2.3)).

\section{Results and discussion}

In this section, we test the present macrotransport theory ((2.2) and (2.10)) by comparing its prediction with that from direct numerical simulation of the two-dimensional transport equations ((2.1) and (2.3)). We solve the macrotransport and the two-dimensional equations using the 'Coefficient Form PDE' and the time-dependent, implicit 'backward differentiation formula (BDF) solver' in COMSOL. The equations are discretized with free 


\section{Macrotransport for diffusiophoretic and chemotactic species}

triangular (for two-dimensional equations) and uniform (for macrotransport equations) elements with a maximum size $5 \times 10^{-3} L$. Adaptive time stepping is selected to capture the flow dynamics on the fast, radial diffusive time scale. Convergence of solutions are acquired with a relative tolerance $\delta=10^{-4}$ between successive solutions. Zero-concentration boundary conditions are set at the channel inlet, $z=0$, and outlet, $z=L$, for both the solute and colloids. In all comparisons below, both solute and colloidal species are sufficiently far away from the inlet and outlet over the course of their time evolution, mimicking an infinitely long channel. Because of the different strengths of hydrodynamic flow examined, different initial locations of the solute and colloid centroid are used across $\S \S 3.1-3.4$. The log-sensing relation is used to model the chemical flow. A small background solute concentration $S_{b}=10^{-3} \max [S(t=0)]$ is imposed to prevent the unphysically large velocity as $S \rightarrow 0$, that is we write $\boldsymbol{u}=M \nabla \log \left(S+S_{b}\right)$. We have tested that, as long as the same $S_{b}$ is used between the macrotransport theory and the direct numerical simulation of the two-dimensional transport equations, it does not alter the excellent agreements between the two sets of results that we will show in $\$ \$ 3.1-3.4$. We focus our analyses on a non-decaying colloidal species, $\Gamma=0$; readers are referred to e.g. Subramanian \& Gill (1974); Shapiro \& Brenner (1986) for detailed discussions of the dispersion of decaying colloids. The averaged solute and colloid concentrations from the two-dimensional equations are obtained from cross-sectionally averaging the (two-dimensional) concentration fields upon solving the equations. In $\S \S 3.1-3.3$, we consider $D_{c} / D_{s}=1$, which is typical for chemotaxis (Ford \& Lauffenburger 1991; Lewus \& Ford 2001; Tindall et al. 2008; Shim et al. 2019). In $\S 3.4$, we consider $D_{c} / D_{s}<1$, which is general to probe diffusiophoresis (Cussler 2009; Shin et al. 2016). In $\$ 3.5$, we discuss the generality of the macrotransport theory to non-log-sensing chemical flows and non-circular channels.

\subsection{Convective axial diffusion regime: weak hydrodynamic flow}

By observing (2.14), for $D_{c} / D_{s}=1$ we choose $\epsilon=5 \times 10^{-3}$ and $P e=1$ to compute the solute and colloidal species transport in the convective axial diffusion regime. Figure 3 shows the time evolution of the normalized averaged solute and colloid concentration profiles. Dotted lines correspond to results obtained from the macrotransport theory, whereas solid lines are from the two-dimensional transport equations. The initial conditions are the same between two sets of results, namely $S /\left.\left(Q_{S} / L\right)\right|_{\hat{t}=0}=$ $\exp \left[-\left(\hat{z} / \hat{f}-\hat{z}_{s} / \hat{f}\right)^{2}\right] /(\hat{f} \sqrt{\pi})$ and $C /\left.\left(Q_{c} / L\right)\right|_{\hat{t}=0}=\exp \left[-\left(\hat{z} / \hat{g}-\hat{z}_{c} / \hat{g}\right)^{2}\right] /(\hat{g} \sqrt{\pi})$, where $Q_{s}$ and $Q_{c}$ are the solute and colloid mass per unit cross-sectional area of the channel, respectively (arbitrary here); $\hat{f}=f / L=0.05$ and $\hat{g}=g / L=0.025$ control the initial width of the solute and colloid distribution, respectively; and $\hat{z}_{s}=z_{s} / L=$ 0.2 and $\hat{z}_{c}=z_{c} / L=0.1$ are the initial location of the solute and colloid centroid, respectively. Since the colloid evolution depends on the solute dynamics, let us first examine figure 3(a). At times longer than the solute radial diffusive time, there is an excellent agreement in the predictions between the macrotransport and the two-dimensional equations. The agreement is supported and quantified by the small difference in the centroid $\Delta \mu_{s}$ (and variance $\Delta \sigma_{s}^{2}$ ) between the two sets of results, where $\Delta \mu_{s} \equiv\left|\left(\int_{0}^{1} z \bar{S} \mathrm{~d} z / \int_{0}^{1} \bar{S} \mathrm{~d} z\right)_{\text {macro }}-\left(\int_{0}^{1} z \bar{S} \mathrm{~d} z / \int_{0}^{1} \bar{S} \mathrm{~d} z\right)_{2 D}\right|$ and $\Delta \sigma_{s}^{2} \equiv$ $\left.\left.\mid\left(\int_{0}^{1} z^{2} \bar{S} \mathrm{~d} z / \int_{0}^{1} \bar{S} \mathrm{~d} z\right)-\mu_{s}^{2}\right)_{\text {macro }}-\left(\int_{0}^{1} z^{2} \bar{S} \mathrm{~d} z / \int_{0}^{1} \bar{S} \mathrm{~d} z\right)-\mu_{s}^{2}\right)_{2 D} \mid$ (Aris 1956; Chu et al. $2020 a$ ). The difference in the centroid of the colloid distribution $\Delta \mu_{c}$ (and variance $\Delta \sigma_{c}^{2}$ ) between the macrotransport and two-dimensional equations, which will be used in 
(a)

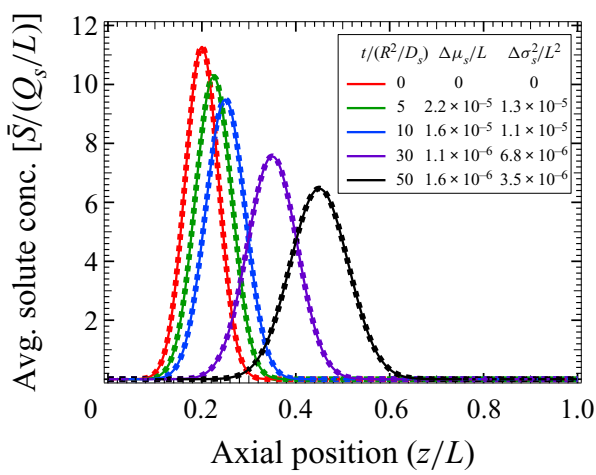

(b)

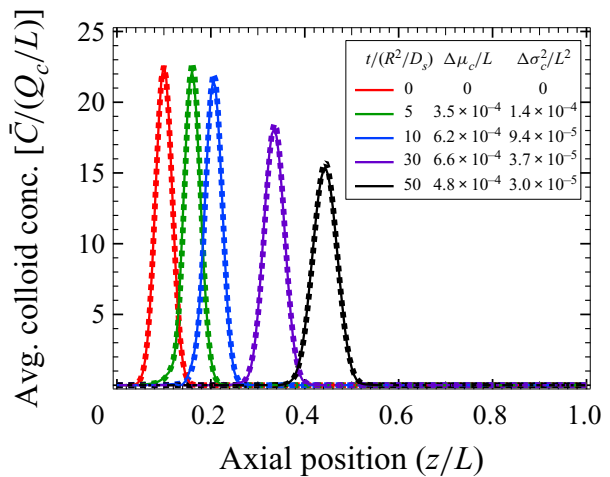

(c)

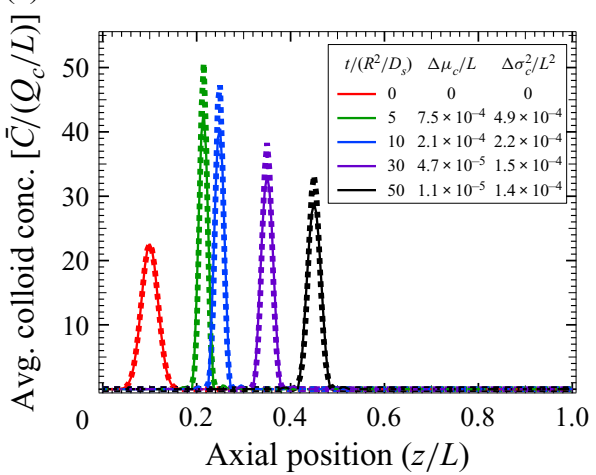

Figure 3. Time evolution of the normalized averaged (a) solute $\bar{S} /\left(Q_{s} / L\right)$ and $(b, c)$ colloidal species $\bar{C} /\left(Q_{c} / L\right)$ concentration profiles for $D_{c} / D_{s}=1, \epsilon=5 \times 10^{-3}$ and $P e=1$. In $(b), M / D_{s}=5$; in $(c), M / D_{s}=25$. Dotted lines: macrotransport theory, (2.2) and (2.10); solid lines: two-dimensional transport equations, (2.1) and (2.3).

figure 3(b,c) and following figures, share a similar definition but with $\bar{S}$ in the integrals replaced by $\bar{C}$.

As the solute distribution evolves in time, so does the solute concentration gradient. This induces a chemical flow of the colloidal species. The evolutions of the colloid concentration profile are shown in figure $3(b, c)$ for $M / D_{s}=5$ and $M / D_{s}=25$, respectively. These values are typical for chemotaxis (Ford \& Lauffenburger 1991; Tindall et al. 2008) where a larger positive $M / D_{s}$ represents a stronger attraction between the solute and the colloid. Such a large $M / D_{s}$ is rare in diffusiophoresis but is potentially attainable in some physico-chemical systems, such as near a liquid-liquid demixing critical point (Sear \& Warren 2017; Ault et al. 2018; Chu et al. 2020a). Let us first inspect figure $3(b)$. We see an excellent agreement between the two sets of results [an equally good agreement is also obtained for $t /\left(R^{2} / D_{s}\right)=1$ but it is not shown here for clarity]. Specifically, by comparing figure $3(a, b)$, colloids are attracted towards the solute. Initially, the separation distance between the solute and colloid centroids is 0.1 . After 50 radial diffusive times, the two centroids coincide. Thus, chemical flow causes a significant colloid movement when hydrodynamic flow is weak and the macrotransport theory captures this attraction response. In fact, a scaling criterion can be obtained from the normalized colloid macrotransport (2.10), which determines when chemical flow is 


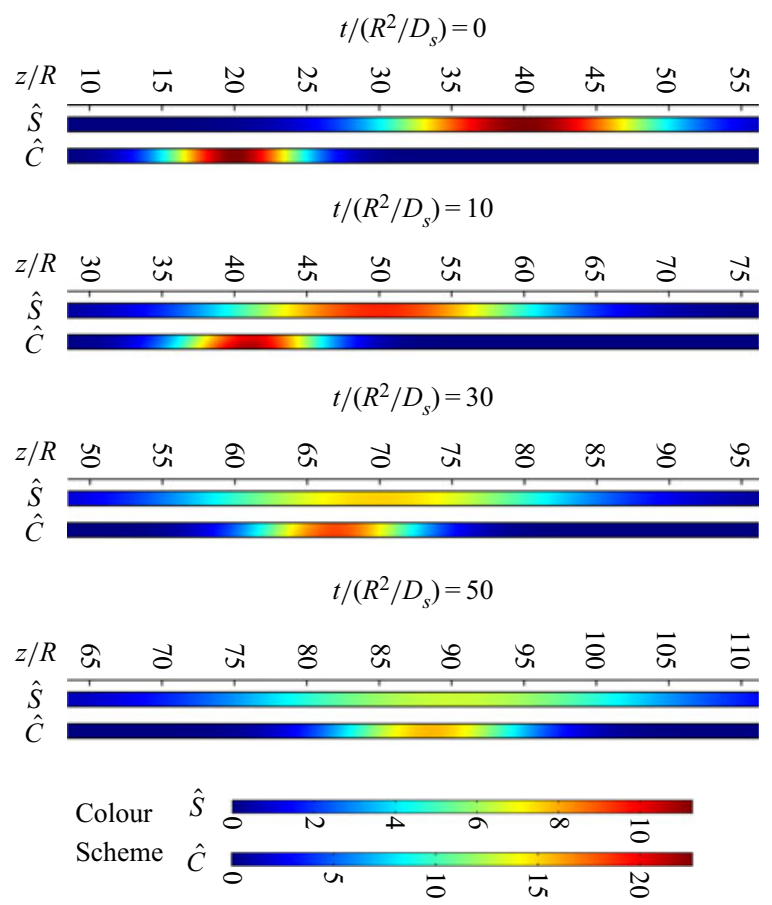

Figure 4. Normalized concentration profiles of the solute $\hat{S}=S /\left(Q_{s} / L\right)$ and colloidal species $\hat{C}=C /\left(Q_{c} / L\right)$ obtained from two-dimensional transport equations, (2.1) and (2.3). Parameters for computation are the same as figure $3(a, b)$.

comparable to the hydrodynamic flow,

$$
\epsilon P e \approx \epsilon^{2} \frac{M}{D_{s}} \frac{1}{\hat{f}} .
$$

The factor $1 / \hat{f}$ arises from the axial length of the solute gradient although the strength of a log-sensing chemical flow is independent of the solute concentration by recognizing that $\partial(\log S) / \partial z=(\partial S / \partial z) / S$. In this case, the strength of the chemical flow, the right-hand side of (3.1), is comparable to the hydrodynamic flow, the left-hand side of the equation.

Figure 4 shows the (two-dimensional) normalized concentration profiles of the solute and colloidal species obtained from the two-dimensional transport equations for figure $3(a, b)$. Since the concentration profiles are axisymmetric about the centreline of the channel, only half of the profile is shown in each contour plot with the top face of the plot corresponding to the centreline of the channel. Hydrodynamic flow is from left to right. In figure 4 , from $\hat{t}=t /\left(R^{2} / D_{S}\right)=0$ to $\hat{t}=10$, the initial solute and colloid distributions are deformed and they follow the parabolic hydrodynamic flow profile. The parabolic profile is more obvious in the colloidal species due to its narrower initial distribution. At $\hat{t}=10$, there is a slight radial non-uniformity in $C$. This is a consequence of the hydrodynamic flow and the non-uniform solute attraction owing to the axially varying solute concentration gradient. Note how the colloid profile spans over different concentration (colour) gradients of the solute distribution. However, the effect of such a slight non-uniformity is insignificant to the macrotransport description, since the predictions of the macrotransport and the two-dimensional equation agree well (figure $3 b$ ). 
(a)

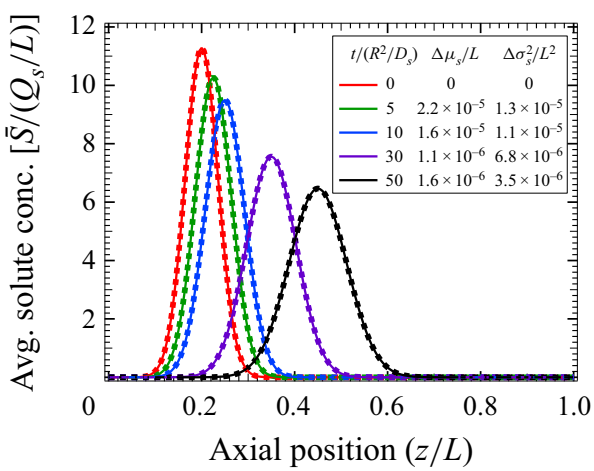

(b)

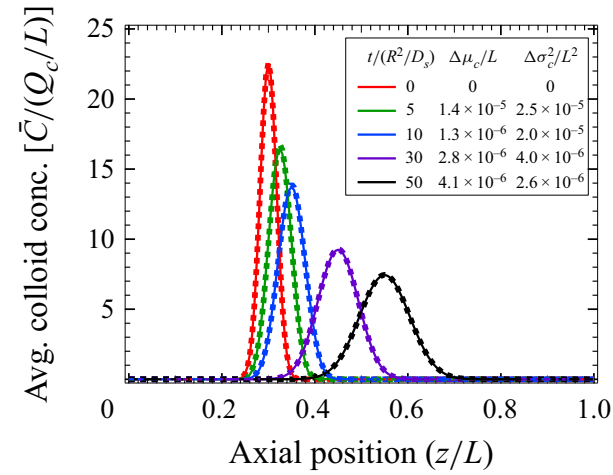

(c)

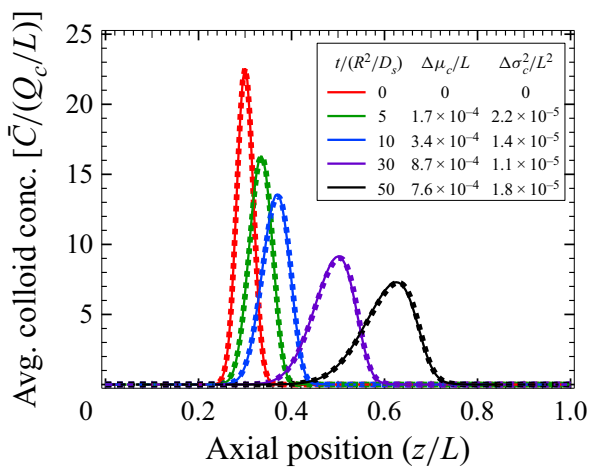

Figure 5. Time evolution of the normalized averaged (a) solute $\bar{S} /\left(Q_{s} / L\right)$ and (b-c) colloidal species $\bar{C} /\left(Q_{c} / L\right)$ concentration profiles for $D_{c} / D_{s}=1, \epsilon=5 \times 10^{-3}$, and $P e=1$. In $(b), M / D_{s}=0$; in $(c), M / D_{s}=-1$. Dotted lines: macrotransport theory, (2.2) and (2.10); solid lines: two-dimensional transport equations, (2.1) and (2.3).

Meanwhile, the colloids are attracted towards the solute with time. As noted in the previous paragraph, the centroids of the solute and the colloid coincide at $\hat{t}=50$.

Next, let us look at figure $3(c)$, where $M / D_{s}=25$ and it shares the same solute evolution figure $3(a)$ with figure $3(b)$. In this case, the colloids are attracted towards the solute much faster compared to figure $3(b)$, as expected. Contraction of the colloid profile is also more prominent. The macrotransport and the two-dimensional equations are in good agreement. The slight deviation between the two sets of prediction is due to numerical artifacts of mass 'leakage' - a consequence of the propagation of discretization errors in solving the convection-dominated transport equation, which is numerically unstable (Ferziger \& Peric 2002). These discretization errors are physically irrelevant and increase with the dimension of the system. This highlights the advantage of using the (one-dimensional) macrotransport equation as opposed to direct numerical simulation. Further, the computational runtime for the macrotransport equation is at least $O\left(10^{3}\right)$ times shorter than the two-dimensional equation.

The macrotransport equation also captures repelling chemical-driven transport, i.e. $M<0$, as shown in figure 5. The solute and colloid initial conditions are the same as before, except that $\hat{z}_{c}=0.3$. On comparing figure $5(b, c)$, the translation of the colloid downstream with chemical-driven repulsion (figure $5 c$ ) is more significant than that without chemical flow figure $5(b)$. In figure $5(c)$, the asymmetry of the colloid distribution 


\section{Macrotransport for diffusiophoretic and chemotactic species}

is due to the fact that, for a Gaussian solute distribution, the log-sensing chemical flow is linearly proportional to the distance from the peak of the solute distribution (Chu et al. 2020a). Thus, in log-sensing chemotaxis/diffusiophoresis, a Gaussian solute distribution induces a spatially non-uniform velocity to the colloid distribution and thus gives rise to an asymmetric distribution. The asymmetry in colloid distribution is not apparent in the attractant case (figure 3) because the colloid distribution is contracted and the non-uniformity in chemical flow is present over a narrow profile only. In contrast, the asymmetric in colloid distribution is more prominent in the repellent case (figure $5 c$ ) because the colloid distribution is broadened and there is a large non-uniformity of chemical flow velocity over the wide profile.

Beyond the above validation, we remark that the convective axial diffusion regime is a quasi-one-dimensional regime of macrotransport that we had implicitly assumed in our previous study of spreading of diffusiophoretic colloids due to transient solute gradients (Chu et al. 2020a). Specifically, the colloids translate with the mean speed of the hydrodynamic flow. Colloid spreading, or macrotransport, is solely governed by the chemical flow and the intrinsic colloid diffusion, while spreading enhancement due to hydrodynamic dispersion is negligible. In other words, our previous study has to observe the rather tight constraint on $P e$ set by (2.14) for the convective axial diffusion regime. Otherwise, for larger $P e$, the hydrodynamic dispersivity becomes comparable to or larger than the intrinsic diffusivity. Then, ignoring dispersivity would yield significant errors, particularly in the variance of the colloid profile.

\subsection{Taylor-Aris regime: intermediately strong hydrodynamic flow}

In this section, we analyse the performance of the macrotransport theory $((2.2)$ and (2.10)) in capturing the two-dimensional transport $((2.1)$ and (2.3)) in the Taylor-Aris regime, where the solute/colloid intrinsic diffusivity is comparable to the corresponding hydrodynamic dispersivity. By observing (2.14), we choose $\epsilon=5 \times 10^{-4}$ and $P e=10$. The solute and colloid initial conditions are the same as figure 3 . As shown in figure 6 , the agreements between the two sets of results are excellent. Distinct from the comparisons in $\$ 3.1$, here the separation between the solute and colloid centroids remain the same over time. This can be understood by referring to the scaling criterion (3.1), where in this case the chemical flow is much weaker than the hydrodynamic flow. Thus, reduction in the separation between the solute and the colloid centroids, which is proportional to the strength of the chemical flow, is negligible compared to the translation due to the hydrodynamic flow.

\subsection{Taylor regime: strong hydrodynamic flow}

In this section, we check the performance of the macrotransport theory in the Taylor regime, where the solute/colloid hydrodynamic dispersivity dominates the corresponding intrinsic diffusivity. From (2.14), we choose $\epsilon=5 \times 10^{-5}$ and $P e=100$. The solute and colloid initial conditions are the same as figure 3 . Figure 7 shows that the predictions of the macrotransport theory agree well with those by the two-dimensional transport equations. As in the Taylor-Aris regime, here the separation between the solute and colloid centroids remain the same over time, since the hydrodynamic flow dominates the chemical flow. In particular, the colloid evolution is essentially identical for $M / D_{s}=0$ and $M / D_{s}=1$. The implication of this observation is that under a strong hydrodynamic flow the solute and colloid equations could be treated as if they were uncoupled. Thus, even though the colloids are chemotactic, their transport due to the solute gradient is negligible. 
(a)

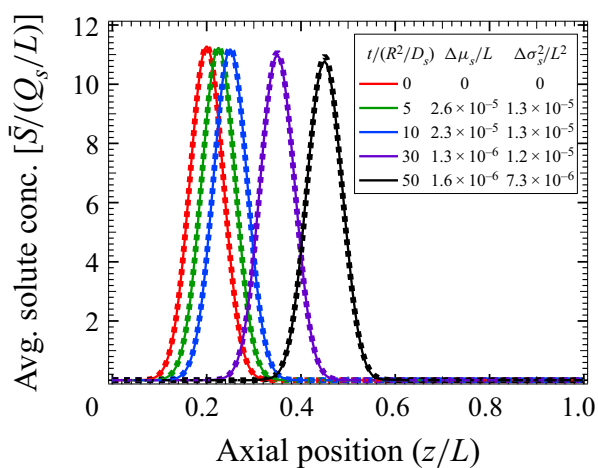

(b)

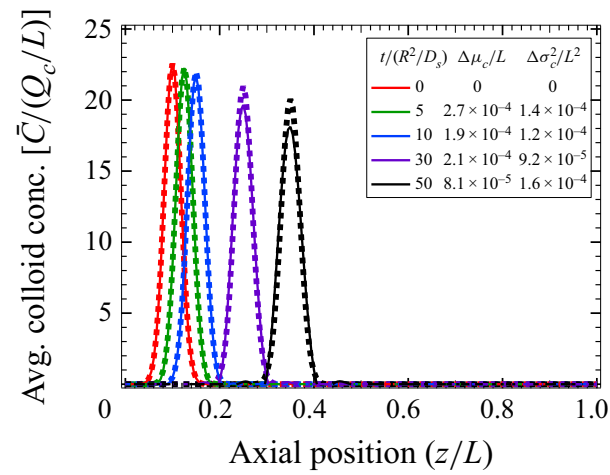

Figure 6. Time evolution of the normalized averaged $(a)$ solute $\bar{S} /\left(Q_{s} / L\right)$ and $(b)$ colloidal species $\bar{C} /\left(Q_{c} / L\right)$ concentration profiles for $D_{c} / D_{s}=1, \epsilon=5 \times 10^{-4}, P e=10$ and $M / D_{s}=1$. Dotted lines: macrotransport theory, (2.2) and (2.10); solid lines: two-dimensional transport equations, (2.1) and (2.3).

(a)

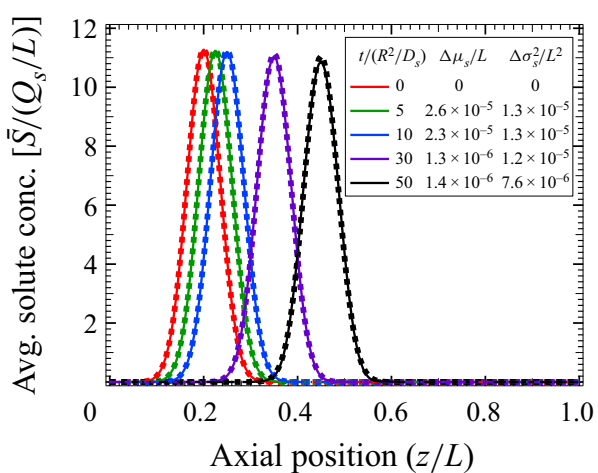

(b)

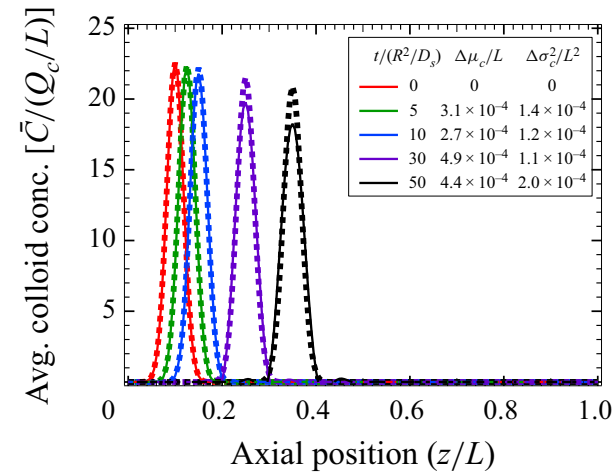

(c)

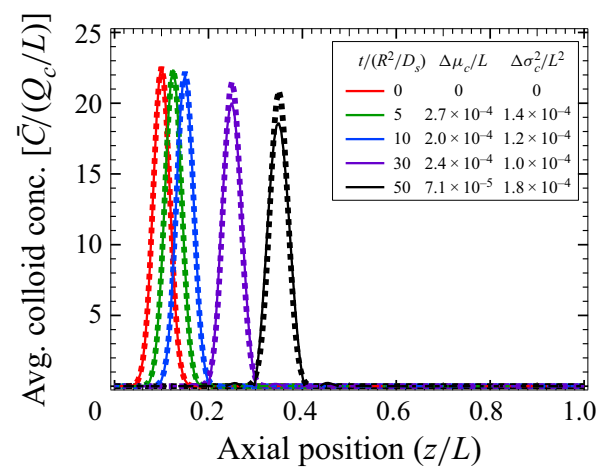

Figure 7. Time evolution of the normalized averaged (a) solute $\bar{S} /\left(Q_{s} / L\right)$ and (b-c) colloidal species $\bar{C} /\left(Q_{c} / L\right)$ concentration profiles for $D_{c} / D_{s}=1, \epsilon=5 \times 10^{-5}$ and $P e=100$. In $(b), M / D_{s}=0$; in $(c), M / D_{s}=1$. Dotted lines: macrotransport theory, (2.2) and (2.10); solid lines: two-dimensional transport equations, (2.1) and (2.3). 
(a)

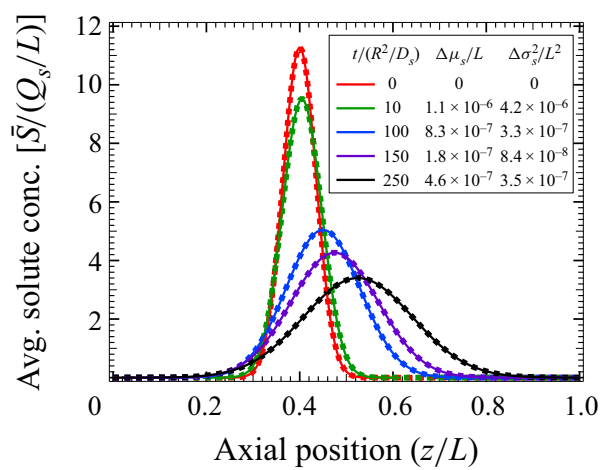

(b)

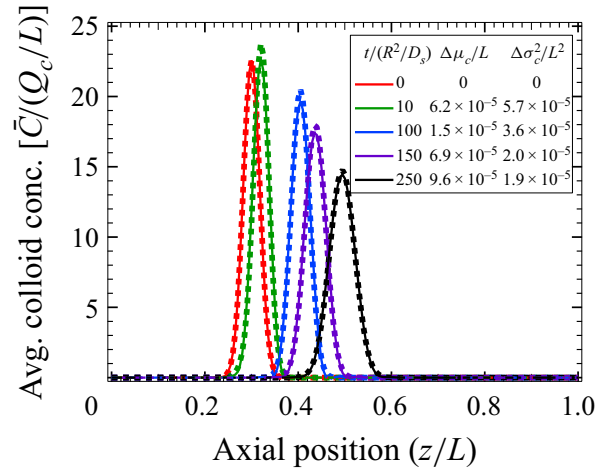

Figure 8. Time evolution of the normalized averaged $(a)$ solute $\bar{S} /\left(Q_{s} / L\right)$ and $(b)$ colloidal species $\bar{C} /\left(Q_{c} / L\right)$ concentration profiles for $D_{c} / D_{s}=0.1, \epsilon=5 \times 10^{-3}, P e=0.1$ and $M / D_{s}=1$. Dotted lines: macrotransport theory, (2.2) and (2.10); solid lines: two-dimensional transport equations, (2.1) and (2.3).

\subsection{Systems with non-unity diffusivity ratio $D_{c} / D_{s}$}

In this section, we test the applicability of the macrotransport theory to diffusiophoresis. Distinct from chemotaxis, the ratio of the colloidal species to solute diffusivity in typical diffusiophoresis settings is much smaller than unity, $D_{c} / D_{s} \leq O\left(10^{-2}\right)$. As a result, probing colloid transport at long times compared to the radial diffusion time scale (where the macrotransport theory applies) demands computing the solute and colloid dynamics for over hundreds of solute radial diffusive times, which is extremely costly. Worse still, the discretization errors, which grow in time and are especially significant in solving the two-dimensional equations, will contaminate the long-time solution and affect the comparison as conducted in the previous sections.

Thus, to show that the macrotransport theory applies to diffusiophoresis and generally for $D_{c} / D_{s} \neq 1$, we perform the comparison for $D_{c} / D_{s}=0.1$, as shown in figure 8 . We conduct the analysis in the convective axial diffusion regime, since we showed that in this regime the effect of the chemical flow is comparable to the hydrodynamic flow. From (2.14), we choose $\epsilon=5 \times 10^{-3}$ and $P e=0.1$. For typical diffusiophoresis, $\left|M / D_{s}\right| \leq 1$ (Prieve et al. 1984; Shin et al. 2016) and we choose $M / D_{s}=1$. The solute and colloid initial conditions are the same as before, except that $\hat{z}_{s}=0.4$ and $\hat{z}_{c}=0.3$. As in the comparisons in the previous sections, the predictions by the macrotransport equations agree very well with those by the two-dimensional transport equations. Substantial attraction of the colloids to the solute is also observed, as expected in the convective axial diffusion regime.

\subsection{Generality of macrotransport to other chemical flows and channel geometries}

In this section, we show how the macrotransport theory can be generalized to channels of other cross-sections and to incorporate non-log-sensing chemical flows. Recall that the macrotransport theory ((2.2), (2.10) and (2.14)) is derived for a uniform circular tube. However, the same macrotransport equations can be applied to channels of other cross-sections by simply replacing the dispersion coefficient 48 by a different coefficient for another channel geometry. For instance, one could easily repeat the averaging procedure in $\S 2$ for a pressure-driven flow in a parallel-plate channel and arrive at the same macrotransport equation, with a dispersion coefficient 210 (Wooding 1960) and 


\section{H.C.W. Chu, S. Garoff, R.D. Tilton and A.S. Khair}

the channel radius $R$ replaced by the channel height $h$. Dispersion coefficients for other cross-sections have been derived, for example concentric/eccentric cylinders as a model for cerebrospinal fluid flows within a spinal cavity (Sankarasubramanian \& Gill 1971; Chu et al. 2020b), non-ideal microchannels with bowing (Dutta, Ramachandran \& Leighton 2006), and channels with slowly varying cross-sections or micropatterns (Bryden \& Brenner 1996; Chu et al. 2019).

The macrotransport theory is also general to incorporate arbitrary forms of chemical flows. As shown in $\$ 2.1$, derivations of the macrotransport equation did not assume any particular form of the chemical flow. While the log-sensing relation is standard to model diffusiophoresis, this relation is just a first approximation to chemotaxis. For example, it does not account for the shear-induced reorientation of chemotactic bacteria, which has been shown to significantly impact bacterial concentration profiles (Rusconi, Guasto \& Stocker 2014; Bearon \& Hazel 2015; Secchi et al. 2020). Chemotactic flow models have been derived in this regard, e.g. (Bearon \& Pedley 2000), and can be incorporated in the macrotransport theory. Other chemotactic velocity relations have been proposed. For instance, Lapidus \& Schiller (1976) introduced the receptor-ligand dissociation constant of the microorganism in their proposed velocity relation, which gives a better agreement with the experiments by Dahlquist, Lovely \& Koshland (1972) and Mesibov, Ordal \& Adler (1973). Rivero et al. (1989) proposed a velocity relation for describing the population-level microorganism transport based on individual microscopic variables, such as the single-cell swim speed, persistence time and temporal receptor occupation. More recently, Menolascina et al. (2017) and Salek et al. (2019) proposed a finite-range log-sensing model, which captures a hallmark of chemotaxis that microorganism log-sensing occurs only above a threshold solute concentration. Thus, the generality of the macrotransport theory to account for arbitrary forms of chemical flows will enable analysis of more biologically realistic models of chemotaxis.

\section{Conclusions}

In this work, we have derived a macrotransport equation for predicting the long-time chemotactic/diffusiophoretic colloidal species transport in a uniform circular tube in which there is a transient solute gradient and the hydrodynamic flow is uniaxial and steady. We have compared the predictions obtained from the macrotransport equation with that from the original, two-dimensional transport equation. The comparisons are conducted in three regimes where classical macrotransport theory applies: convective axial diffusion (weak hydrodynamic flow); Taylor-Aris (intermediately strong hydrodynamic flow); and Taylor (strong hydrodynamic flow) regime. In all three regimes, we have obtained excellent agreements between the macrotransport and two-dimensional equations. Discretization errors, which increase with the dimension of the problem, are mitigated in the reduced-order macrotransport model and the computational runtime for solving the macrotransport model is in general $O\left(10^{3}\right)$ times shorter than numerical solution of the two-dimensional advection-diffusion-reaction equation. We have identified that, in the convective axial diffusion regime, hydrodynamic dispersion is negligible compared to intrinsic diffusion. Thus, the evolution of the cross-sectionally averaged colloid concentration can be described by the macrotransport equation where colloid spreading is solely due to the chemical flow and the intrinsic colloid diffusion. In addition, we have presented a scaling criterion for determining when a chemical flow would cause significant colloid transport amid a hydrodynamic flow. We have also discussed how the macrotransport equation can be generalized for channels of arbitrary cross-section as well 
as to incorporate other forms of chemical flows necessary for capturing recently revealed hallmarks of chemotaxis.

For future work, the macrotransport theory can be employed to examine in more detail the interplay between chemical and hydrodynamic flows on colloid transport. A few relevant studies exist (Yan, Bouwer \& Hilpert 2014; Yan \& Hilpert 2014) although their scopes are restricted due to the high computational cost associated with fully resolving the two-/three-dimensional solute and colloid transport. The present theory can be leveraged to efficiently explore the large parameter space involved in a system with hydrodynamic and chemical flows, particularly comparing predictions with different velocity models proposed for chemotaxis. For instance, in the macrotransport model an exact solution can be obtained for deposition of a point source of colloid in a Gaussian distribution of solute, which we will describe in detail in future work. This seemingly idealized example is in fact applicable to examine the long-time colloid transport with arbitrary forms of initial distribution, since any distribution asymptotes to a Gaussian at long times (Bender \& Orszag 1999; Chu et al. 2020a).

Acknowledgements. H.C.W.C. acknowledges the assistance of T.-L. Hsieh in setting up the COMSOL simulations.

Funding. This work was partly funded by Procter \& Gamble. H.C.W.C. also acknowledges the startup funding support from the Division of Sponsored Programs, Department of Chemical Engineering, and Herbert Wertheim College of Engineering at University of Florida.

Declaration of interest. The authors report no conflict of interest.

\section{Author ORCIDs.}

(i) Henry C.W. Chu https://orcid.org/0000-0002-2705-0863;

(1) Stephen Garoff https://orcid.org/0000-0001-8148-8535;

(1) Robert D. Tilton https://orcid.org/0000-0002-6535-9415;

(1) Aditya S. Khair https://orcid.org/0000-0001-6076-2910.

\section{REFERENCES}

Abecassis, B., Cottin-Bizonne, C., Ybert, C., Ajdari, A. \& Bocquet, L. 2008 Boosting migration of large particles by solute contrasts. Nat. Mater. 7, 785-789.

AdAdevoh, J.S.T., Ostvar, S., Wood, B. \& Ford, R.M. 2017 Modeling transport of chemotactic bacteria in granular media with distributed contaminant sources. Environ. Sci. Technol. 51, 14192-14198.

ADLER, J. 1966 Chemotaxis in bacteria. Science 12, 708-716.

ANDERSON, J.L. 1989 Colloid transport by interfacial forces. Annu. Rev. Fluid Mech. 21, 61-99.

ARIS, R. 1956 On the dispersion of a solute in a fluid flowing through a tube. Proc. R. Soc. Lond. A $235,67-77$.

Ault, J.T., Shin, S. \& Stone, H.A. 2018 Diffusiophoresis in narrow channel flows. J. Fluid Mech. $854,420-448$.

Ault, J.T., Warren, P.B., Shin, S. \& Stone, H.A. 2017 Diffusiophoresis in one-dimensional solute gradients. Soft Matt. 13, 9015-9023.

BAILEY, H.R. \& GOGARTY, W.B. 1962 Numerical and experimental results on the dispersion of a solute in a fluid in laminar flow through a tube. Proc. R. Soc. Lond. A 269, 352-367.

BALU, B. \& KhAIR, A.S. 2018 Role of Stefan-Maxwell fluxes in the dynamics of concentrated electrolytes. Soft Matt. 14, 8267-8275.

Banerjee, A., Williams, I., Azevedo, R.N., Helgeson, M.E. \& Squires, T.M. 2016 Soluto-inertial phenomena: designing long-range, long-lasting, surface-specific interactions in suspensions. Proc. Natl Acad. Sci. USA 113, 8612-8617.

BEARON, R.N. \& HAZEL, A.L. 2015 The trapping in high-shear regions of slender bacteria undergoing chemotaxis in a channel. J. Fluid Mech. 771, R3.

Bearon, R.N. \& Pedley, T.J. 2000 Modeling run-and-tumble chemotaxis in a shear flow. Bull. Math. Biol. 62, 775-791. 


\section{H.C.W. Chu, S. Garoff, R.D. Tilton and A.S. Khair}

Bender, C.M. \& OrszaG, S.A. 1999 Advanced Mathematics Methods for Scientists and Engineers. Springer-Verlag.

Brenner, H. \& EdwARds, D.A. 1993 Macrotransport Processes. Butterworth-Heinemann.

Brown, D.A. \& Berg, H.C. 1974 Temporal stimulation of chemotaxis in Escherichia coli. Proc. Natl Acad. Sci. USA 71, 1388-1393.

BRYDEN, M.D. \& BRENNER, H. 1996 Multiple-timescale analysis of Taylor dispersion in converging and diverging flows. J. Fluid Mech. 311, 343-359.

Chu, H.C.W., Garoff, S., Przybycien, T.M., Tilton, R.D. \& Khair, A.S. 2019 Dispersion in steady and time-oscillatory two-dimensional flows through a parallel-plate channel. Phys. Fluids 31 , 022007.

Chu, H.C.W., Garoff, S., Tilton, R.D. \& Khair, A.S. 2020 a Advective-diffusive spreading of diffusiophoretic colloids under transient solute gradients. Soft Matt. 16, 238-246.

Chu, H.C.W., Garoff, S., Tilton, R.D. \& Khair, A.S. $2020 b$ Dispersion in steady and time-oscillatory flows through an eccentric annulus. AIChE J. 66, e16831.

Cussler, E.L. 2009 Diffusion: Mass Transfer in Fluid Systems. Cambridge University Press.

Dahlquist, F.W., Lovely, P. \& Koshland, D.E. 1972 Qualitative analysis of bacterial migration in chemotaxis. Nat. New Biol. 236, 120-123.

DutTa, D., RAmachandran, A. \& Leighton, D.T. 2006 Effect of channel geometry on solute dispersion in pressure-driven microfluidic systems. Microfluid Nanofluid 2, 275-290.

Eisenbach, M., Lengeler, J.W., Varon, M., Gutnick, D., Meili, R., Firtel, R.A., Segall, J.E., Omann, G.M., TAmada, A. \& Murakami, F. 2004 Chemotaxis. Imperial College Press.

ENGELmAnN, T.W. 1881 Neue methode zur untersuchung der sauerstoffaussheidung pflanzlicher und thierischer organismen. Pflugers Arch. Gesamte Physiol. Menschen Tiere 25, 285-292.

FERZIGer, J.H. \& PERIC, M. 2002 Computational Methods for Fluid Dynamics. Springer-Verlag.

Ford, R.M. \& CUMMINGS, P.T. 1992 On the relationship between cell balance equations for chemotaxis cell populations. SIAM J. Appl. Maths 52, 1426-1441.

FORD, R.M. \& HARVEY, R.W. 2007 Role of chemotaxis in the transport of bacteria through saturated porous media. Adv. Water Resour. 30, 1608-1617.

FORD, R.M. \& LAUFFENBURGER, D.A. 1991 Measurement of bacterial random motility and chemotaxis coefficients: II. Application of single-cell-based mathematical model. Biotechnol. Bioengng 37, 661-672.

Gill, W.N. \& Sankarasubramanian, R. 1970 Exact analysis of unsteady convective diffusion. Proc. R. Soc. Lond. A 316, 341-350.

Golding, I., Kozlovsky, Y., Cohen, I. \& Ben-JaCOB, E. 1998 Studies of bacterial branching growth using reaction-diffusion models for colonial development. Phys. A 260, 510-554.

GuPTA, P.S. \& GuPTA, A.S. 1972 Effect of homogeneous and heterogeneous reactions on the dispersion of a solute in the laminar flow between two plates. Proc. R. Soc. Lond. A 330, 59-63.

Kalinin, Y.V., JiAng, L., TU, Y. \& WU, M. 2009 Logarithmic sensing in Escherichia coli bacterial chemotaxis. Biophys. J. 96, 2439-2448.

Kar, A., Chiang, T., Rivera, I.O., Sen, A. \& Velegol, D. 2015 Enhanced transport into and out of dead-end pores. ACS Nano. 9, 746-753.

Keller, E.F. \& SEgEL, L.A. 1971 Traveling bands of chemotactic bacteria: a theoretical analysis. J. Theor. Biol. 30, 235-248.

LAPIDUS, I.R. \& SCHILlER, R. 1976 Model for the chemotactic response of a bacterial population. Biophys. J. 16, 779-789.

LEWUS, P. \& FORD, R.M. 2001 Quantification of random motility and chemotaxis bacterial transport coefficients using individual-cell and population-scale assays. Biotechnol. Bioengng 75, 292-304.

MARBACH, S. \& BoCQUet, L. 2019 Osmosis, from molecular insights to large-scale applications. Chem. Soc. Rev. 48, 3102-3144.

MARX, R.B. \& AitKen, M.D. 2000 Quantification of chemotaxis to naphthalene by Pseudomonas putida G7. Appl. Environ. Microbiol. 65, 2847-2852.

Menolascina, F., Rusconi, R., Fernandez, V.I., Smriga, S., Aminzare, Z., Sontag, E.D. \& Stocker, R. 2017 Logarithmic sensing in Bacillus subtilis aerotaxis. Syst. Biol. Appl. 3, 16036.

Mesibov, R., Ordal, G.W. \& AdLER, J. 1973 The range of attractant concentrations for bacterial chemotaxis and the threshold and size of response over this range. J. Gen. Physiol. 62, 203-223.

NG, C.O. \& Rudraiah, N. 2008 Convective diffusion in steady flow through a tube with a retentive and absorptive wall. Phys. Fluids 20, 073604.

Palacci, J., Abecassis, B., Cottin-Bizonne, C., Ybert, C. \& BocQuet, L. 2010 Colloidal motility and pattern formation under rectified diffusiophoresis. Phys. Rev. Lett. 104, 138302. 
Palacci, J., Cottin-Bizonne, C., Ybert, C. \& Bocquet, L. 2012 Osmotic traps for colloids and macromolecules based on logarithmic sensing in salt taxis. Soft Matt. 8, 980-994.

PARKinson, J.S. \& Kofoid, E.C. 1992 Communication modules in bacterial signaling proteins. Annu. Rev. Genet. 26, 71-112.

Peraud, J., Nonaka, A.J., Bell, J.B., Donev, A. \& Garcia, A.L. 2017 Fluctuation-enhanced electric conductivity in electrolyte solutions. Proc. Natl Acad. Sci. USA 114, 10829-10833.

Prieve, D.C, Anderson, J.L., Ebel, J.P. \& Lowell, M.E. 1984 Motion of a particle generated by chemical gradients. Part 2. Electrolytes. J. Fluid Mech. 148, 247-269.

ProbsteIn, R.F. 2003 Physicochemical Hydrodynamics: An Introduction. Wiley-Interscience.

Raynal, F., Bourgoin, M., Cottin-Bizonne, C., Ybert, C. \& Volk, R. 2018 Advection and diffusion in a chemically induced compressible flow. J. Fluid Mech. 847, 228-243.

Raynal, F. \& VolK, R. 2019 Diffusiophoresis, batchelor scale and effective Peclet number. J. Fluid Mech. 876, 818-829.

Rivero, M.A., Tranquillo, R.T., Buettner, H.M. \& Lauffenburger, D.A. 1989 Transport models for chemotactic cell populations based on individual cell behavior. Chem. Engng Sci. 44, 2881-2897.

Rivero-Hudec, M. \& LAufFenburger, D.A. 1986 Quantification of bacterial chemotaxis by measurement of model parameters using the capillary assay. Biotechnol. Bioengng 28, 1178-1190.

Rusconi, R., Guasto, J.S. \& Stocker, R. 2014 Bacterial transport suppressed by fluid shear. Nat. Phys. 10, 212-217.

Salek, M.M., Carrara, F., Fernandez, V., Guasto, J.S. \& Stocker, R. 2019 Bacterial chemotaxis in a microfluidic T-maze reveals strong phenotypic heterogeneity in chemotactic sensitivity. Nat. Commun. $10,1877$.

SANKARASUbramaniAn, R. \& GiLl, W.N. 1971 Taylor diffusion in laminar flow in an eccentric annulus. Intl J. Heat Mass Transfer 14, 905-919.

SEAR, R.P. 2019 Diffusiophoresis in cells: a general nonequilibrium, nonmotor mechanism for the metabolism-dependent transport of particles in cells. Phys. Rev. Lett. 122, 128101.

SEAR, R.P. \& WARREN, P.B. 2017 Diffusiophoresis in nonadsorbing polymer solutions: the Asakura-Oosawa model and stratification in drying films. Phys. Rev. E 96, 062602.

Secchi, E., Vitale, A., Mino, G., Kantsler, V., Eberl, L., Rusconi, R. \& Stocker, R. 2020 The effect of flow on swimming bacteria controls the initial colonization of curved surfaces. Nat. Commun. $11,2851$.

SEGEL, L.A. 1977 A theoretical study of receptor mechanisms in bacterial chemotaxis. SIAM J. Appl. Maths $32,653-665$.

Servais, P., Billen, G. \& Rego, J.V. 1985 Rate of bacterial mortality in aquatic environments. Appl. Environ. Microbiol. 49, 1448-1454.

ShAPIRO, M. \& BRENNER, H. 1986 Taylor dispersion of chemically reactive species: irreversible first-order reactions in bulk and on boundaries. Chem. Engng Sci. 41, 1417-1433.

Shi, N., Nery-AZEvedo, R., AbDel-FATtAh, A.I. \& SQuires, T.M. 2016 Diffusiophoretic focusing of suspended colloids. Phys. Rev. Lett. 117, 258001.

Shim, S., Stone, H.A. \& Ford, R.M. 2019 Chemotaxis in shear flow: similarity solutions of the steady-state chemoattractant and bacterial distributions. AIChE J. 65, e16713.

Shin, S., Ault, J.T., Warren, P.B. \& Stone, H.A. 2017 Accumulation of colloidal particles in flow junctions induced by fluid flow and diffusiophoresis. Phys. Rev. X 4, 041038.

Shin, S., Um, E., SAbass, B., Ault, J.T., Rahimi, M., Warren, P.B. \& Stone, H.A. 2016 Size-dependent control of colloid transport via solute gradients in dead-end channels. Proc. Natl Acad. Sci. USA 113, 257-261.

STAFFELD, P.O. \& QuinN, J.A. 1989 Diffusion-induced banding of colloid particles via diffusiophoresis: 1. Electrolytes. J. Colloid Interface Sci. 130, 69-87.

Subramanian, R.S. \& Gill, W.N. 1974 Dispersion models of unsteady tubular reactors. Can. J. Chem. Engng. 52, 563-568.

TAYLOR, G.I. 1953 Dispersion of soluble matter in solvent flowing slowly through a tube. Proc. R. Soc. Lond. A 219, 186-203.

Tindall, M.J., Porter, S.L., Maini, P.K., Gaglia, G. \& Armitage, J.P. 2008 Overview of mathematical approaches used to model bacterial chemotaxis I: the single cell. Bull. Math. Biol. 70, 1525-1569.

Valdes-Parada, F.J., Porter, M.L., Narayanaswamy, K., Ford, R.M. \& Wood, B.D. 2009 Upscaling microbial chemotaxis in porous media. Adv. Water Resour. 32, 1413-1428. 
Velegol, D., Garg, A., Guha, R., Kar, A. \& Kumar, M. 2016 Origins of concentration gradients for diffusiophoresis. Soft Matt. 12, 4686-4703.

VIDYANIDHI, V. \& MURTY, M.S. 1976 The dispersion of a chemically reacting solute in a micropolar fluid. Intl J. Engng Sci. 14, 1127-1133.

WAdhams, G.H. \& ARmitage, J.P. 2004 Making sense of it all: bacterial chemotaxis. Nature 5, 1024-1037.

WoODING, R.A. 1960 Instability of a viscous liquid of variable density in a vertical Hele-Shaw cell. J. Fluid Mech. 7, 501-515.

Wu, M., Roberts, J.W., Kim, S., KосH, D.L. \& DeLisA, M.P. 2006 Collective bacterial dynamics revealed using a three-dimensional population-scale defocused particle tracking technique. Appl. Environ. Microbiol. 72, 4987-4994.

YAN, Z., BOUWER, E.J. \& HiLPERT, M. 2014 Coupled effects of chemotaxis and growth on traveling bacterial waves. J. Contam. Hydrol. 164, 138-152.

YAN, Z. \& HiLPERT, M. 2014 A multiple-relaxation-time Lattice-Boltzmann model for bacterial chemotaxis: effects of initial concentration, diffusion, and hydrodynamic dispersion on traveling bacterial bands. Bull. Math. Biol. 76, 2449-2475. 